\title{
Toward Sportomics: Shifting From Sport Genomics to Sport Postgenomics and Metabolomics Specialties. Promises, Challenges, and Future Perspectives
}

Together with experience, training, dietary intake, and other environmental factors, the biological and genetic makeup of an athlete play a major role in exercise physiology in terms of performance and outcomes. ${ }^{1}$ Sport genomics has shown that some DNA singlenucleotide polymorphisms can be associated with athlete level and performance (such as elite/world-class athletic status), having an impact on physical activity-related variables like endurance; strength; sprint; power; speed; flexibility; energetic expenditure; neuromuscular coordination; and respiratory, metabolic, and cardiorespiratory fitness, among others. Moreover, single-nucleotide polymorphisms have been shown to correlate with other parameters, including psychological traits. ${ }^{2}$ The athletic phenotype is extremely complex and multifactorial, depending on the combination of different features and characteristics. ${ }^{3}$ On this basis, sport performance is a "complex science," like that of metadata and multiomics profiles.

Several ambitious projects (like the Exercise at the LimitInherited Traits of Endurance [ELITE], GAMES, Gene Skeletal Muscle Adaptive Response to Training or Gene SMART, GENATHLETE, Genetics of Elite Status in Sport or GENESIS, 1000 Athlomes, Super-Athletes, and POWERGENE trials) are aimed at discovering genomics-based biomarkers with an adequate predictive power. ${ }^{4}$ These projects are aimed at overcoming the major drawbacks that plagued previous investigations, generally relying on small and rather heterogeneous cohorts of athletes. Sport genomics could enable researchers, athletes, sport scientists, and coaches/managers to optimize and maximize physical performance and identify prevention strategies in the field of individual risk of sport-related injuries (like Achilles tendinopathy or rotator cuff pathologies). ${ }^{3}$

However, the athlete genome is only a pebble in the mosaic of sport physiology. ${ }^{3}$ Exercise has a profound impact also on the human proteome, for instance, finely tuning ATP-related pathways and mitochondrial protein synthesis, as well as proteins belonging to inflammation, antioxidation, anticoagulation, and iron. ${ }^{5}$ Moreover, exercise modulates transcription patterns and epigenetics, as well as metabolic profiles. All these different omics specialties (like sport genomics, epigenomics, transcriptomics, proteomics, and metabolomics/metabonomics) converge in a unique approach termed as "sportomics." 3,6 Introduced for the first time by Brazilian scientist Cameron and colleagues, the word "metabolomics" can be defined as a holistic and top-down framework, characterizing all non-hypothesis-framed but data-driven research for systematically uncovering an individual's biomolecular changes during exercise and sport. ${ }^{6}$

Sportomics includes both genomics and postgenomics specialties and, comprehensively relying on the "athlete's biological passport" or profile, would enable the systematic study of sportinduced responses and adaptations at any level (genome, transcriptome, proteome, etc). ${ }^{3}$ This is the ambitious goal of the large collaborative initiative "Athlome Project Consortium," as stated in the "Santorini Declaration" during the symposium held in Greece in May 2015. Pursuing this goal would definitively pave the way for a personalized, individualized understanding of the orchestrated effects of physical activity. ${ }^{4}$

Among others, sport metabolomics is of particular importance since, unlike genes and proteins, the function of which is dependent on epigenetic changes and posttranslational modifications, metabolites are the direct result of biochemical interactions and are, therefore, powerful and reliable factors in physiological studies. ${ }^{3}$ Metabolites are produced as the end products of chemical processes and are considered the final result of gene expression. Changes in the metabolome occur in the timescale of seconds or minutes and exactly reflect the physiological status of the body at a certain time. ${ }^{3}$ Quintas et $\mathrm{al}^{7}$ used metabolomics to study the relationship(s) between internal and external load indicators during a football season and reported that steroid hormone biosynthesis and metabolism, and tyrosine and tryptophan metabolism pathways were the main external load indicators in football. Furthermore, another study correlated endurance performance with a list of metabolites, which were involved in the energy metabolism, antioxidant defense, cell damage, and central nervous system-signaling metabolites. ${ }^{8}$ In another study, Al-Khelaifi et al ${ }^{9}$ studied resting blood samples of 4 elite athletes' categories (high and moderate endurance, high- and moderate-power athletes) and reported that high-power and high-endurance athletes showed a different metabolome, mainly associated with steroid biosynthesis, fatty acid metabolism, oxidative stress, and energy-related pathways. This study has opened a new insight into sport talent identification.

However, according to a recently published systematic review of the studies in the field of sport metabolomics/metabonomics, most researchers have focused on prolonged exercise practice/programs, while the effects of acute exercise bouts were generally overlooked, with a few notable exceptions. ${ }^{10}$ If these gaps are properly acknowledged and addressed, sportomics could be highly relevant for sport sciences. Indeed, it could provide athletes, sport managers/coaches, and other relevant actors and stakeholders with detailed information concerning personalized training and nutrition, potentially allowing them to (1) identify talents, (2) enhance/optimize performance, (3) design ad hoc training and conditioning programs, and (4) minimize the risk of injuries and therefore contribute to optimizing each athlete's own potential.

Nicola Luigi Bragazzi, York University, Canada Kayvan Khoramipour (k.khoramipour@kmu.ac.ir), Kerman University of Medical Science, Iran Anis Chaouachi, Center of Sports Medicine, Tunisia Karim Chamari, IJSPP Associate Editor, ASPETAR, Qatar Orthopedic and Sports Medicine Hospital, Qatar 


\section{References}

1. Gabriel BM, Zierath JR. The limits of exercise physiology: from performance to health. Cell Metab. 2017;25(5):1201-1202. PubMed ID: 28467920 doi:10.1016/j.cmet.2017.04.018

2. Ahmetov II, Fedotovskaya ON. Current progress in sports genomics. In: Advances in Clinical Chemistry (Vol. 70, pp. 247-314). Amsterdam, the Netherlands: Elsevier; 2015.

3. Hoffman NJ. Omics and exercise: global approaches for mapping exercise biological networks. Cold Spring Harb Perspect Med. 2017; 7(10):a029884. PubMed ID: 28348175 doi:10.1101/cshperspect. a029884

4. Pitsiladis YP, Tanaka M, Eynon N, et al. Athlome Project Consortium: a concerted effort to discover genomic and other "omic" markers of athletic performance. Physiol Genomics. 2016;48(3):183-190. PubMed ID: 26715623 doi:10.1152/physiolgenomics.00105.2015

5. Lanza IR, Sreekumaran Nair K. Regulation of skeletal muscle mitochondrial function: genes to proteins. Acta Physiol. 2010;199(4): 529-547. PubMed ID: 20345409 doi:10.1111/j.1748-1716.2010.02124.x
6. Bassini A, Cameron LC. Sportomics: building a new concept in metabolic studies and exercise science. Biochem Biophys Res Commun. 2014;445(4):708-716. PubMed ID: 24406165 doi:10.1016/j. bbrc.2013.12.137

7. Quintas G, Reche X, Sanjuan-Herráez JD, et al. Urine metabolomic analysis for monitoring internal load in professional football players. Metabolomics. 2020;16(4):45. PubMed ID: 32222832 doi:10.1007/ s11306-020-01668-0

8. Monnerat G, Sánchez CAR, Santos CGM, et al. Different signatures of high cardiorespiratory capacity revealed with metabolomic profiling in elite athletes. Int J Sports Physiol Perform. 2020;15(8):11561167. PubMed ID: 32335533 doi:10.1123/ijspp.2019-0267

9. Al-Khelaifi F, Diboun I, Donati F, et al. A pilot study comparing the metabolic profiles of elite-level athletes from different sporting disciplines. Sports Med Open. 2018;4(1):2. PubMed ID: 29305667 doi:10.1186/s40798-017-0114-z

10. Contrepois K, Wu S, Moneghetti KJ, et al. Molecular choreography of acute exercise. Cell. 2020;181(5):1112-1130.e16. PubMed ID: 32470399 doi:10.1016/j.cell.2020.04.043 